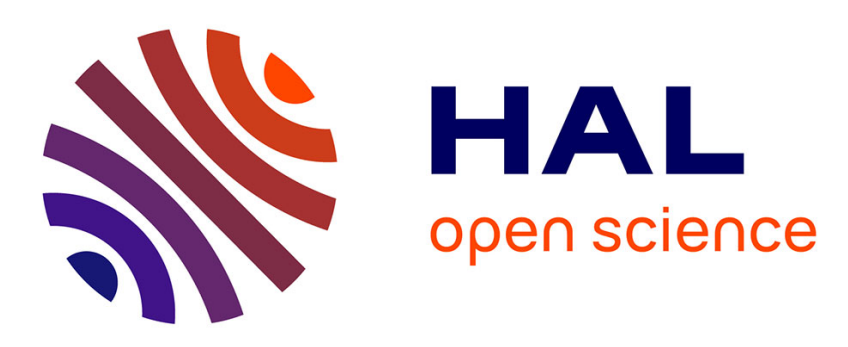

\title{
Sparse Method for Tip-Timing Signals Analysis with Non Stationary Engine Rotation Frequency
}

Antoine Bouchain, José Picheral, Elisabeth Lahalle, Agathe Vercoutter, Arnaud Talon

\section{- To cite this version:}

Antoine Bouchain, José Picheral, Elisabeth Lahalle, Agathe Vercoutter, Arnaud Talon. Sparse Method for Tip-Timing Signals Analysis with Non Stationary Engine Rotation Frequency. 26th European Signal Processing Conference (EUSIPCO 2018), Sep 2018, Rome, Italy. hal-01896808

\section{HAL Id: hal-01896808 \\ https://hal-centralesupelec.archives-ouvertes.fr/hal-01896808}

Submitted on 16 Oct 2018

HAL is a multi-disciplinary open access archive for the deposit and dissemination of scientific research documents, whether they are published or not. The documents may come from teaching and research institutions in France or abroad, or from public or private research centers.
L'archive ouverte pluridisciplinaire HAL, est destinée au dépôt et à la diffusion de documents scientifiques de niveau recherche, publiés ou non, émanant des établissements d'enseignement et de recherche français ou étrangers, des laboratoires publics ou privés. 


\title{
Sparse Method for Tip-Timing Signals Analysis with Non Stationary Engine Rotation Frequency
}

\author{
Antoine Bouchain \\ José Picheral \\ Elisabeth Lahalle \\ Laboratoire des Signaux et Systèmes \\ CentraleSupélec - CNRS - Université Paris Sud - Université Paris Saclay \\ 3 Rue Joliot-Curie, 91192 Gif sur Yvette, France \\ Email:name.surname@centralesupelec.fr
}

\author{
Agathe Vercoutter \\ Arnaud Talon \\ Safran Helicopter Engines \\ Avenue Joseph Szydlowski, 64510 Bordes, France \\ Email: name.surname@safrangroup.com
}

\begin{abstract}
Blades vibrations must be measured in operations to validate blade design. Tip-timing is one of the classical measurement methods but its main drawback is the generation of sub-sampled and non-uniform sampled signals. This paper presents a new sparse method for tip-timing spectral analysis that makes use of engine rotation variations. Assuming that blade vibration signals yield to line spectra, a sparse signal model is introduced as a linear system. The solution to the problem is obtained by ADMM (Alternating Direction Method of Multipliers) with a $\ell^{1}$-regularization. Results for simulated and real signals are given to illustrate the efficiency of this method. The main advantages of the proposed method are to provide a fast solution and to take into account the variations of the rotation speed. Results show that this approach reduces frequency aliasings caused by the low sampling frequency of the measured signals.
\end{abstract}

\section{INTRODUCTION}

Compressors and turbines blades vibrations are a key element in the turbomachinery test certification campaign. Indeed, in operating conditions, blades are excited by alternating aerodynamic turbulences that may cause mechanical resonances. Compressors and turbines test aim at identifying and quantifying blade mechanical responses under such excitation sources. Blades vibrations are traditionally measured by strain gauges. However, due to the high implementation cost and complexity, only a few blades are generally instrumented. Besides, sticking a gauge and its wire on a blade slightly modifies its mechanical response. All these gauges drawbacks made popular an other measurement method, less intrusive and easier to set up, called tip-timing. The acquisition system is composed by a set of $C$ optical probes mounted on the stator and monitoring the blade tip passing times, see Fig.1. When a blade is passing in front of the laser beam, the optical probe detects the blade and records the corresponding time. This time can vary if the blade is vibrating and the delay can be interpreted as a blade tip displacement, knowing the rotation frequency. As shown Fig.1, the sampling times, indicated by the sampling function $e(t)$, are irregular because probes are non-equidistant on the stator. Moreover, the irregular sampling pattern is repeated at each revolution, then this time sampling pattern is irregular and periodic. Thus, the frequency sampling

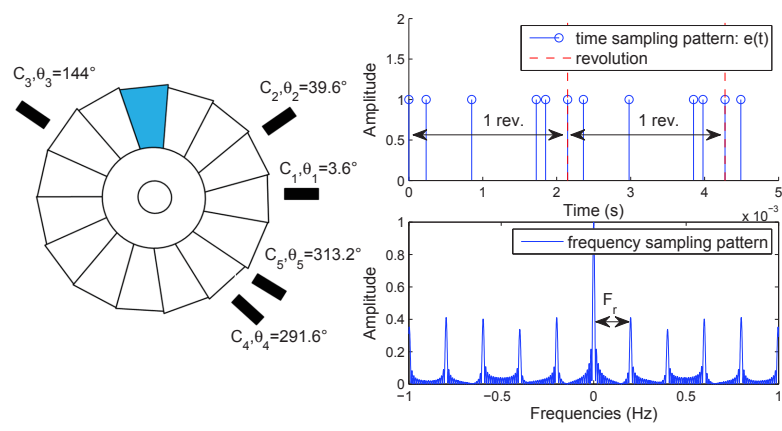

Fig. 1. Blade vibration measurement with tip-timing technology.

pattern consists of peaks located at every multiple of the rotation frequency. Most often, the spectral frequency components are largely higher than the rotation frequency. Consequently, signals are sub-sampled and the spectrum contains aliased components generated by the periodic sampling patterns.

For the last 30 years, several solutions have been developed to face these difficulties [1]-[3]. But with those methods, the multi-components spectral analysis remains delicate. Furthermore, most of them lie on strong hypotheses about blade mechanical behavior, which are not necessarily confirmed in real functioning. Recently, new approaches that do not require such hypotheses have been proposed. Minimum Variance Spectral Estimator (MVSE) [4], is an iterative method to estimate the covariance matrix for Capon method [5] in the case of tip-timing signals. Nevertheless, this method involves a high computation time. A compressed sensing approach has been developed in [6] based on a Sparse Reconstruction Method (denoted as SRM in this paper). An other $\ell^{1}$-regularized method, developped in [7] by the authors, has been proposed for spectral analysis of tip-timing signals. Note that, inherent hypothesis of [6] and [7] methods do not allow engine speed variations. Consequently, on real data, the rotation speed variations limit the length of the observation window and can lead to erroneous amplitudes estimations. To this day, to our knowledge, no method using engine speed variations has been developed for tip-timing analysis. Therefore, this paper 
presents a new fast sparse method that makes use of the engine rotation variations to eliminate artefacts in tip timing spectral analysis. The use of sparsity in the method is justified with the a priori knowledge that blades present a limited number of vibrations modes on the engine range of use. Thus, the vibration signal spectrum consists of a very limited number of frequency lines. As far as line spectra of non uniform sampled signals are concerned, they have already been studied with sparse methods (see [8], for instance). Nonetheless, in the context of tip-timing signals, the issue is slightly different since the sampling pattern, see Fig. 1, leads to numerous aliasings artifacts in the signal spectrum on the considered frequency range.

This paper is organized as follow: section II introduces the tip-timing signal model and the proposed estimator. Section III exposes the experimental results and performances in terms of mean squared error (MSE), spectrum sparsity and computation time. Finally, section IV concludes the paper and discusses future studies.

\section{SPARSE MODELS OF TIP-TIMING SIGNAL}

Assuming that the vibration signal of a unique blade is $x(t)$, the measured signal $y(t)$ is extracted from a set of $C$ probes by revolution over $N_{t}$ revolutions, with non stationary rotation frequency $F_{r}(n)=1 / T_{r}(n)$, where $1 \leq n \leq N_{t}$ is the revolution number. The model of the time continuous sampled signal over $N$ points is:

$$
y(t)=\sum_{k=1}^{N} x\left(t_{k}\right) \delta\left(t-t_{k}\right)
$$

Besides, probes are placed around the stator at arbitrary angular positions $\theta_{c}, 1 \leq c \leq C$. Sampling pattern is then irregular. For a given revolution $n$, the sampling times are:

$$
t_{k}=\theta_{c} T_{r}(n) / 2 \pi+\sum_{i=1}^{n-1} T_{r}(i)
$$

with $k=c+C(n-1)$.

Vibrations are supposed to be sinusoidal and frequencybounded on $\left[0, f_{\max }\right]$. The signal $x(t)$ is then approximated by $\xi(t)$ such as $x(t)=\xi(t)+b(t)$, where $b(t)$ includes the model error and the noise measurement. The model $\xi(t)$ can be written as a sum of $M$ cosines whose frequency components are $f_{m}=\frac{m}{M} f_{\max }$ of respective amplitudes $\alpha_{m}$ and phases $\varphi_{m}$ :

$$
\xi(t)=\sum_{m=1}^{M} \alpha_{m} \cos \left(2 \pi f_{m} t+\varphi_{m}\right)
$$

and its Fourier transform is given by:

$$
\begin{aligned}
\tilde{\xi}(f)= & \frac{1}{2} \sum_{m=1}^{M} \alpha_{m} e^{j \varphi_{m}} \delta\left(f-f_{m}\right) \\
& +\alpha_{m} e^{-j \varphi_{m}} \delta\left(f+f_{m}\right) .
\end{aligned}
$$

Blades are only vibrating on a limited number of modes, hence, most of the amplitudes $\alpha_{m}$ are zero. This particular sparsity property about the $\alpha_{m}$ will be exploited further. The signal model presented in this section leads to two sampling models: the model of irregular periodic sampling (abbreviated as MIPS) which uses the $F_{r}$ periodicity and the presented model of absolute sampling (abbreviated as MASS) when $F_{r}$ is not constant.

\section{A. Model of Irregular Periodic Sampling: MIPS}

The model of irregular periodic sampling has been developed in [7] and some concepts are recalled here. Assuming the rotating frequency is not varying during runs, the sampling is periodic of period $T_{r}=T_{r}(n), \forall 1 \leq n \leq N_{t}$. Thus, the sampled vibration signal can be written as:

$$
y(t)=x(t) e(t)
$$

with $e(t)$ the sampling function. This function can be written as the convolution between a Dirac comb of period $T_{r}$, noted $\amalg_{T_{r}}(t)$, and a tip-timing sampling pattern $e_{T T}(t)$ linked to the irregular probe distribution:

$$
\begin{aligned}
e(t) & =e_{T T}(t) * \amalg_{T_{r}}(t) \\
& =\sum_{c=1}^{C} \delta\left(t-\theta_{c} T_{r} / 2 \pi\right) * \sum_{n=-\infty}^{+\infty} \delta\left(t-n T_{r}\right) .
\end{aligned}
$$

A spectral estimation of $x(t)$ is given by the Non Uniform Fourier Transform (NUFT) $\tilde{y}(f)=\sum_{k=1}^{N} x\left(t_{k}\right) e^{-j 2 \pi f t_{k}}$ of $y(t)$ and can be modeled by:

$$
\tilde{\zeta}(f)=F_{r} \sum_{n=0}^{+\infty}\left(\tilde{\xi} * \tilde{\Pi}_{\left[0, N_{t} T_{r}\right]}\right)\left(f-n F_{r}\right) \tilde{e}_{T T}\left(n F_{r}\right) .
$$

This spectrum is the convolution of $\tilde{\xi}(f)$ with the observation window spectrum $\tilde{\Pi}(f)$ and replicated on the multiples of the rotation frequency. Unlike the regular case, each refolding is weighted by $\tilde{e}_{T T}\left(n F_{r}\right)$, related to the probes positions. With (3b) and an even number of revolutions, (6) can be rewritten as:

$$
\tilde{\zeta}(f)=\sum_{m=1}^{M} a_{m} g_{m}^{+}(f)+a_{m}^{*} g_{m}^{-}(f)
$$

where

$$
\begin{aligned}
g_{m}^{ \pm}(f)= & \frac{N_{t}}{2} e^{-j 2 \pi f \frac{N_{t}}{F_{r}}} \sum_{n=0}^{\infty} \tilde{e}_{T T}\left(n F_{r}\right) \\
& \times \operatorname{sinc}\left(\frac{N_{t}}{F_{r}}\left(f \pm f_{m}-n F_{r}\right)\right) e^{-j \pi\left( \pm f_{m}\right) \frac{N_{t}}{F_{r}}} \\
a_{m}= & \alpha_{m} e^{j \varphi_{m}}
\end{aligned}
$$

and $a_{m}^{*}$ the complex conjugate of $a_{m}$. By splitting the quantities into real and imaginary parts, respectively marked with the exponent ${ }^{R}$ and ${ }^{I}$, (7) becomes:

$$
\begin{gathered}
\tilde{\zeta}^{R}(f)=\sum_{m=1}^{M} a_{m}^{R}\left(g_{m}^{+R}+g_{m}^{-R}\right)(f)+a_{m}^{I}\left(-g_{m}^{+I}+g_{m}^{-I}\right)(f) \\
\tilde{\zeta}^{I}(f)=\sum_{m=1}^{M} a_{m}^{R}\left(g_{m}^{+I}+g_{m}^{-I}\right)(f)+a_{m}^{I}\left(g_{m}^{+R}-g_{m}^{-R}\right)(f)
\end{gathered}
$$


With the interval $I$ discretised into $\left(f_{k}\right), 1 \leq k \leq N_{f}$ values, (7) can then be written as a linear system:

$$
\begin{aligned}
\tilde{\boldsymbol{\zeta}} & =\boldsymbol{G} \boldsymbol{\theta} \\
& =\left[\begin{array}{ll}
G_{1} & G_{2} \\
G_{3} & G_{4}
\end{array}\right] \boldsymbol{\theta}
\end{aligned}
$$

where

$$
\tilde{\boldsymbol{\zeta}}=\left[\tilde{\zeta}^{R}\left(f_{1}\right) \ldots \tilde{\zeta}^{R}\left(f_{N_{f}}\right) \tilde{\zeta}^{I}\left(f_{1}\right) \ldots \tilde{\zeta}^{I}\left(f_{N_{f}}\right)\right]^{T}
$$
of size $\left[2 N_{f} \times 1\right]$,

$$
\begin{aligned}
& \boldsymbol{G}_{\mathbf{1}}=\left[\left(g_{M}^{+R}+g_{M}^{-R}\right)\left(f_{k}\right) \ldots\left(g_{1}^{+R}+g_{1}^{-R}\right)\left(f_{k}\right)\right], \\
& \boldsymbol{G}_{\mathbf{2}}=\left[\left(-g_{1}^{+I}+g_{1}^{-I}\right)\left(f_{k}\right) \ldots\left(-g_{M}^{+I}+g_{M}^{-I}\right)\left(f_{k}\right)\right], \\
& \boldsymbol{G}_{\mathbf{3}}=\left[\left(g_{M}^{+I}+g_{M}^{-I}\right)\left(f_{k}\right) \ldots\left(g_{1}^{+I}+g_{1}^{-I}\right)\left(f_{k}\right)\right], \\
& \boldsymbol{G}_{\mathbf{4}}=\left[\left(g_{1}^{+R}-g_{1}^{-R}\right)\left(f_{k}\right) \ldots\left(g_{M}^{+R}-g_{M}^{-R}\right)\left(f_{k}\right)\right]
\end{aligned}
$$

of sizes $[N f \times M]$,

$$
\boldsymbol{\theta}=\left[\begin{array}{llllll}
a_{M}^{R} & \ldots & a_{1}^{R} & a_{1}^{I} & \ldots & a_{M}^{I}
\end{array}\right]^{T} \text { of size }[2 M \times 1],
$$

and $[.]^{T}$ is the transposition operator. Since $\left\{f_{m}\right\}_{m=1, \ldots, M}$ are usually choosen to sample uniformly the frequency range of interest, $\boldsymbol{\theta}$ provides an estimation of the amplitudes $a_{m}$ of each $f_{m}$ and thus a spectral representation of the signal. In the case of line spectrum and from the (3b) comment, most of the $a_{m}$ are null so that $\boldsymbol{\theta}$ is sparse. This motivate us to introduce sparsity prior in the regularization term. In order to solve (8) with sparse a priori, a $\ell^{1}$-norm penalisation has been introduced on $\boldsymbol{\theta}$, with a regularisation parameter $\lambda$. Consequently, a LASSO [9] problem is obtained:

$$
J(\boldsymbol{\theta})=\min _{\boldsymbol{\theta}}\|\tilde{\boldsymbol{y}}-\boldsymbol{G} \boldsymbol{\theta}\|_{2}^{2}+\lambda\|\boldsymbol{\theta}\|_{1} .
$$

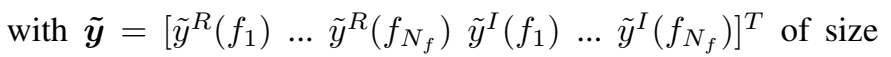
$\left[2 N_{f} \times 1\right]$. Criterion (9) is solved with the Alternating Direction Method of Multipliers (ADMM) [10] algorithm.

\section{B. Proposed model : Model of AbSolute Sampling, MASS}

The MIPS previously mentioned is no longer accurate in the case of non stationary rotation frequency. Thus, in this paper we propose a generalized model called Model of AbSolute Sampling (MASS), that takes into account the real rotation frequency fluctuations. On the considered observation windows, the frequencies $f_{m}$ are supposed asynchronous and are neither rotation frequency nor time dependent. With (1) and (3a), the sampled vibration signal is rewritten as follows:

$$
\begin{aligned}
y(t) & =\sum_{m=1}^{M} a_{m}^{R} \sum_{k=1}^{N} \cos \left(2 \pi f_{m} t\right) \delta\left(t-t_{k}\right) \\
& -\sum_{m=1}^{M} a_{m}^{I} \sum_{k=1}^{N} \sin \left(2 \pi f_{m} t\right) \delta\left(t-t_{k}\right)+b(t) .
\end{aligned}
$$

Similarly to (8), a linear system is obtained:

$$
\boldsymbol{y}=\boldsymbol{E} \boldsymbol{\theta}+\boldsymbol{b}
$$

where

$$
\begin{aligned}
& \boldsymbol{y}=\left[\begin{array}{llll}
y\left(t_{1}\right) & y\left(t_{2}\right) \ldots y\left(t_{N}\right)
\end{array}\right]^{T} \text { of size }[N \times 1], \\
& \boldsymbol{b}=\left[\begin{array}{llll}
b\left(t_{1}\right) & b\left(t_{2}\right) & \ldots & b\left(t_{N}\right)
\end{array}\right]^{T} \text { of size }[N \times 1], \\
& \boldsymbol{E}=\left[\begin{array}{ll}
\boldsymbol{E}^{\boldsymbol{c}} & \boldsymbol{E}^{\boldsymbol{s}}
\end{array}\right] \text {, }
\end{aligned}
$$

where $\boldsymbol{E}_{n, m}^{\boldsymbol{c}}=\cos \left(2 \pi f_{M-m+1} t_{n}\right)$ and $\boldsymbol{E}_{n, m}^{\boldsymbol{s}}=$ $-\sin \left(2 \pi f_{m} t_{n}\right), \boldsymbol{E}_{\boldsymbol{c}}$ and $\boldsymbol{E}_{\boldsymbol{s}}$ are both of sizes $N \times M$ and $\boldsymbol{\theta}$ remains the same as in (8). To solve (11), a LASSO problem is obtained:

$$
P(\boldsymbol{\theta})=\min _{\boldsymbol{\theta}}\|\boldsymbol{y}-\boldsymbol{E} \boldsymbol{\theta}\|_{2}^{2}+\lambda\|\boldsymbol{\theta}\|_{1} .
$$

Criterion (12) is also solved with the ADMM [10] algorithm. The MASS model is more generic than the MIPS one. In consequence, when the speed rotation is constant, the main difference is due to the size of the matrices $\boldsymbol{E}$ and $\boldsymbol{G}$ but the results are quite similar.

\section{EXPERIMENTAL RESULTS}

As a reminder, due to the irregular sub-sampling, spectrum consists of numerous aliased components that require adapted methods. Such methods must enable to distinguish real components among aliased ones and to estimate their amplitude accurately, with reasonable computation times. The following section presents the estimation performances of the proposed method in terms of MSE, frequency identification capacity and computation time.

\section{A. Synthetic signal}

Let us consider a synthetic signal denoted as "Signal A" whose characteristics are coherent in comparison to real test cases. It is composed of three frequency components, $f_{1}^{A}=$ $213 \mathrm{~Hz}, f_{2}^{A}=1626 \mathrm{~Hz}$ and $f_{3}^{A}=1931 \mathrm{~Hz}$. A white Gaussian noise is added whose variance $\sigma^{2}$ is:

$$
\sigma^{2}=\sigma_{f_{2}^{A}}^{2} 10^{-S N R / 10},
$$

where the signal to noise ratio (SNR) is $-10 d B$ and with $\sigma_{f_{2}^{A}}^{2}$ the power of $f_{2}^{A}$ component. The signal is generated with $C^{2}=5$ probes at angular position $3.6^{\circ}, 39.6^{\circ}, 144^{\circ}, 291.6^{\circ}$ and $313.2^{\circ}$ during $N_{t}=200$ revolutions (the signal duration is $0.4 s$ ). Rotation frequency is established with a $p=12$ autoregressive (AR) process [11] to which an acceleration trend is added $\left(\delta_{F_{r}}=0.005 \mathrm{~Hz}\right.$ by revolution) starting from $F_{r}=470 \mathrm{~Hz}$ to end at $471 \mathrm{~Hz}$. It is important to notice that $f_{1}^{A}$ aliasings will be located on $f_{2}^{A}$ since $f_{2}^{A}-f_{1}^{A}=3 \times 471 H z$.

The spectral estimation provided by the proposed method (based on MASS) is compared with the MVSE estimations in Fig.2. The MIPS and SRM spectra are also given in Fig.2. The regularization parameter for the LASSO problem is set to $\lambda=1$. MVSE was set with 5 iterations with 3 windows of 199 revolutions. For SRM, 359 spots and $10^{-10}$ as threshold were used. To represent the frequency range, $[0,2499] \mathrm{Hz}$, a grid of $M=2500$ points is used with a step of $1 \mathrm{~Hz}$. Note that $1 \mathrm{~Hz}$ step is a sufficient resolution for this application, since blades physical responses are spread among a few hertz. The estimated amplitudes are summarized Table I. MASS and MVSE methods are quite similar in terms of amplitude estimation. As expected in the rotation variation context, MIPS and SRM fail: on MIPS spectrum, numerous aliasings are not removed and amplitudes are largely underestimated; on SRM spectrum, one component is missing and estimations are more erroneous than MASS and MVSE. 

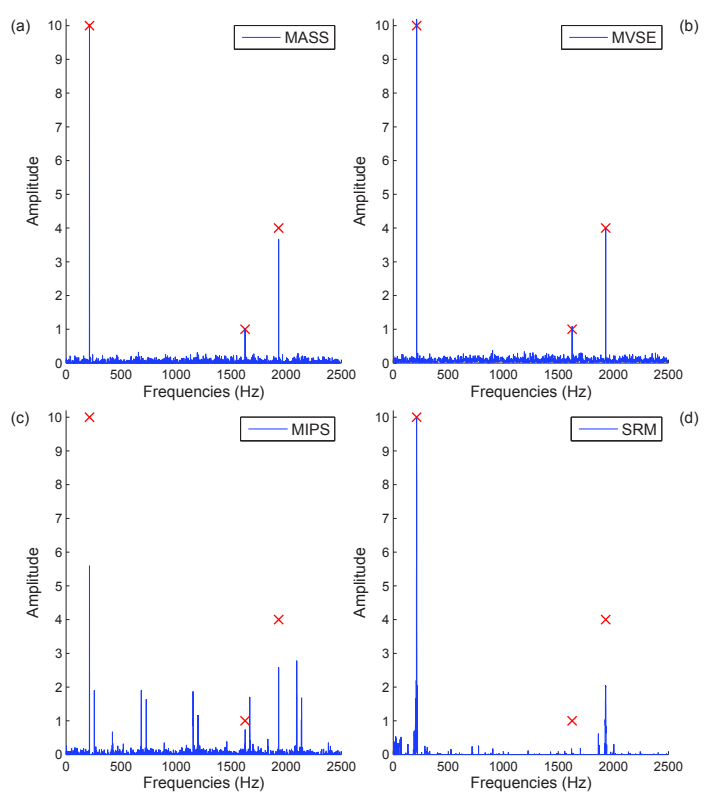

Fig. 2. Signal A spectra with MASS (a), MVSE (b), MIPS (c) and SRM (d).

TABLE I

AMPLITUDE ESTIMATIONS.

\begin{tabular}{|c||c||c||c|}
\cline { 2 - 4 } \multicolumn{1}{l||}{} & $f_{1}^{A}=213 \mathrm{~Hz}$ & $f_{2}^{A}=1626 \mathrm{~Hz}$ & $f_{3}^{A}=1931 \mathrm{~Hz}$ \\
\hline \hline Theory & 10 & 1 & 4 \\
\hline MASS & 10.05 & 1.02 & 3.67 \\
\hline MVSE & 10.19 & 1.09 & 3.99 \\
\hline MIPS & 5.60 & 0.29 & 2.58 \\
\hline SRM & 9.76 & 0 & 1.99 \\
\hline
\end{tabular}

The tests have been conducted in terms of MSE and spectrum sparsity for 100 realizations of Signal A with various signal durations. MSE is calculated separately for each frequency component and results are given Fig.3. In the legend, the subscripts indexes, after the name of the method, indicate the number of revolutions used. Due to excessive computation time, MVSE has been tested only for 100 and 200 revolutions. MVSE has better performances than MASS when comparing them for the same number of revolutions. Nevertheless, increasing the number of samples improves the MASS performances. With MASS, a large number of revolutions can be exploited because the model takes into account the rotation speed variations allowing an increase of the observation window. For 500 revolutions, the MSE of MASS is slightly better than the one of MVSE over 200 revolutions, especially for $f_{2}^{A}$ component despite $f_{1}^{A}$ aliasings.

The performances of the frequency components identification are estimated by $S_{\tilde{y}}$, which is the $\ell^{2}$-norm of the spectrum background (ie. by removing the three frequency components) and expressed as follows:

$$
S_{\tilde{y}}=\sqrt{\sum_{m=1}^{M}\left|\hat{a}_{m}\right|^{2}-\sum_{k=1}^{3}\left|\hat{a}_{m_{k}}\right|^{2}}
$$

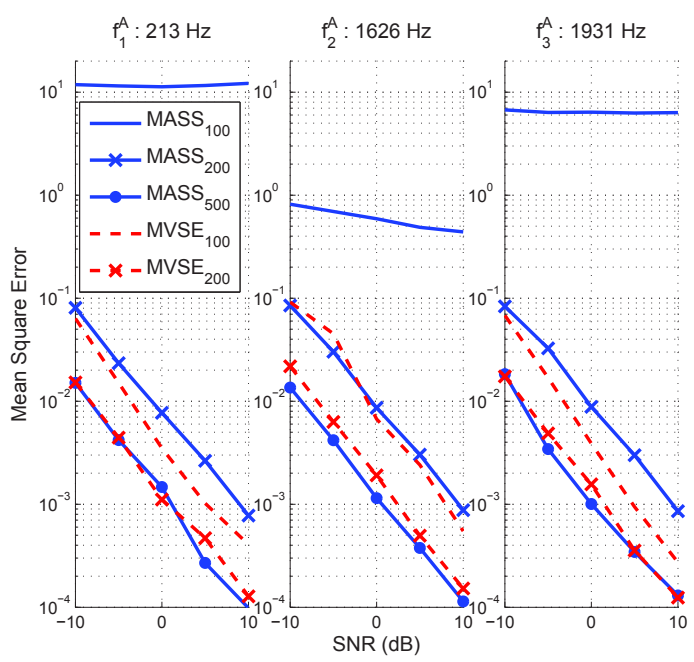

Fig. 3. MSE of MASS and MVSE for 3 components.

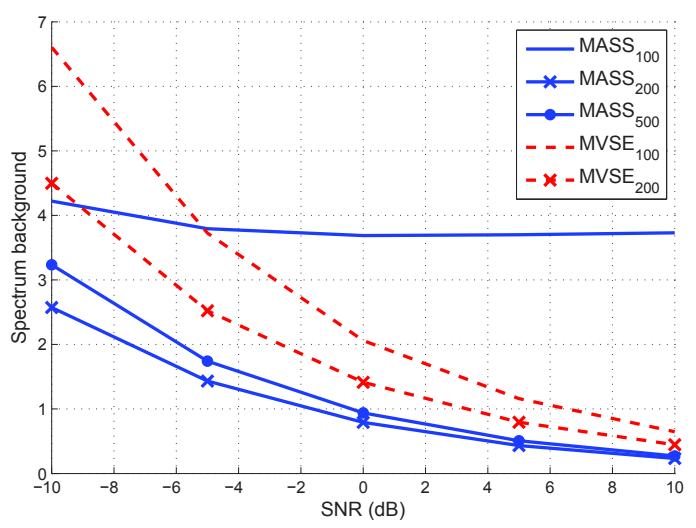

Fig. 4. Spectrum background of MASS and MVSE

where $\hat{a}_{m}$ is the estimation of $a$ and $m_{k}$ the index of the frequency components $\left\{f_{k}^{A}\right\}_{k=1,2,3}$ of the simulated signals. Results are given Fig.4. As expected, spectrum background decreases when SNR increases. Furthermore, the MVSE spectrum is less sparse than the MASS one. Beyond a certain number of revolutions, 200 in this example, the MASS spectrum background does not decrease anymore but slightly increases. Consequently, a compromise must be found between sparsity and estimation accuracy.

The different execution time of MASS, MVSE and MIPS has been calculated and data are summarized Table II. They have been gathered under MATLAB ${ }^{\circledR}$ with a four core Intel ${ }^{\circledR}$ Core i7 processor unit cadenced at $2.5 \mathrm{GHz}$. It can be observed that the execution time of MASS increases practically linearly with the number of revolutions whereas it increases exponentially for MVSE. Notice that MASS $_{500}$ is about 10 times faster than $\mathrm{MVSE}_{200}$ and provides more accurate estimation. As for MIPS, its time decreasing is due to its number of iterations falling. Nevertheless, MASS is unquestionably the fastest method whatever the number of revolutions. 
TABLE II

COMPUTATION TIME (IN SECONDS) FOR MASS, MVSE AND MIPS.

\begin{tabular}{|c||c|c|c|}
\hline Method & 100 & 200 & 500 \\
\hline \hline MASS & 0.8 & 1.7 & 5.5 \\
\hline MVSE & 11 & 48 & 361 \\
\hline MIPS & 11.4 & 10.7 & 9.5 \\
\hline
\end{tabular}

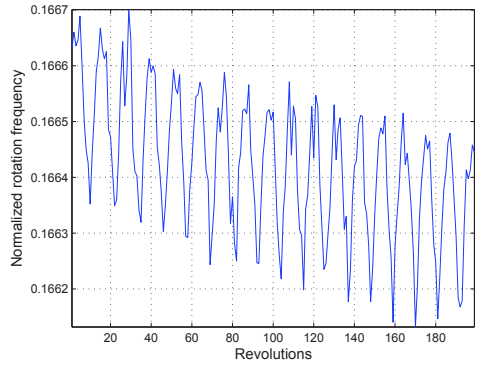

Fig. 5. Engine rotation frequency of Signal B.

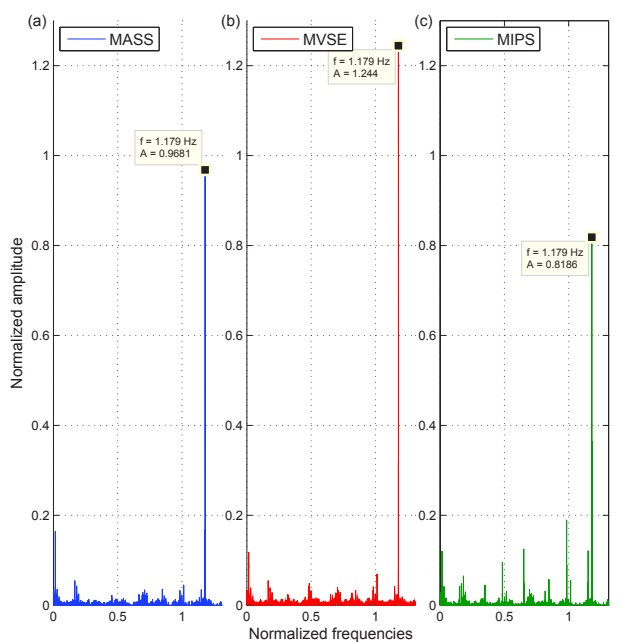

Fig. 6. Signal B spectra with ADMM-MASS (a), MVSE (b) and ADMMMIPS (c).

\section{B. Real signal}

The real signal, referenced as "Signal B" is extracted from data of an experimental compressor test rig. Signal B was recorded over 200 revolutions and is composed of a unique frequency component at 1.179 . Fig.5 shows the fluctuation on the rotation frequency and the results of the methods are given Fig.6 with normalized amplitudes. For ADMM, the regularization parameter is set to $\lambda=10^{-4}$. MVSE was set with 3 windows of 199 revolutions over 5 iterations. The frequency range, $[0,3999] \mathrm{Hz}$, is computed with $M=4000$. Similarly to synthetic signals, MIPS presents numerous aliased components and is not well adapted to the signal due to the rotation frequency variations. MASS and MVSE both provide an estimate of the frequency component but with about $20 \%$ difference on estimated amplitudes.
Regarding the execution time, MASS took $4 s$ and MIPS $33 s$ with $K_{M A S S}=K_{M I P S}=228$ iterations whereas MVSE took 89 s with $K_{M V S E}=5$ iterations. Comparing to signal $\mathrm{A}$, the time difference is due to the increase of the range frequency. However for MASS, this increase is negligible and MASS is about twenty times faster than MVSE. It can be explained by the complexity of the algorithms which are $O\left(2 M N^{2}+4 K_{M A S S}\left(M N+N^{2}\right)+N^{3} / 3\right)$ for MASS and $O\left(\left(4 K_{M V S E}+1\right) M N^{2}+6 K_{M V S E} N^{3}\right)$ for MVSE.

\section{CONCLUSION}

The non stationarity of the rotation speed needs to be taken into account for spectral analysis of tip-timing signals. Taking the rotation speed into consideration allows the presented method to extend the observation windows, which is an essential element to reduce aliased components and to improve amplitudes estimation on the spectra. The proposed method yields promising results. For long observation windows, it performs as well as the acknowledged method MVSE and even surpasses it in terms of spectrum background sparsity and MSE with a much lower computation time. Its quasi-linearity with the revolution number makes it very fast compared to previous methods. Some perspectives are currently investigated about the choice of the minimization algorithm as well as the optimization of the probes angular positions.

\section{REFERENCES}

[1] S. Heath and M. Imregun, "An improved single parameter method for turbomachinery blade vibration measurements using optical laser probes," Journal of Mechanical Science, pp. 1047-1058, 1996.

[2] M. Zielinski and G. Ziller, "Noncontact vibration measurements on compressor rotor blades," Meas. Sci. Technol., vol. 11, no. 7, p. 847, 2000.

[3] S. Heath and M. Imregun, "A Survey of Blade Tip-Timing Measurement Techniques for Turbomachinery Vibration," J. Eng. Gas Turbines Power, vol. 120 , no. 4, pp. 784-791, Oct. 1998.

[4] A. Vercoutter, M. Berthillier, A. Talon, B. Burgardt, and J. Lardies, "Tip Timing Spectral Estimation Method For Aeroelastic Vibrations of Turbomachinery Blades," p. 9, 2011.

[5] J. Capon, "High-resolution frequency-wavenumber spectrum analysis," Proceedings of the IEEE, vol. 57, no. 8, pp. 1408-1418, Aug. 1969.

[6] J. Lin, Z. Hu, Z.-S. Chen, Y.-M. Yang, and H.-L. Xu, "Sparse reconstruction of blade tip-timing signals for multi-mode blade vibration monitoring," Mechanical Systems and Signal Processing, vol. 81, pp. 250-258, Dec. 2016.

[7] A. Bouchain, J. Picheral, E. Lahalle, and A. Vercoutter, "Méthode parcimonieuse pour l'analyse spectrale de signaux tip-timing," Sep. 2017.

[8] S. Bourguignon, H. Carfantan, and J. Idier, "A Sparsity-Based Method for the Estimation of Spectral Lines From Irregularly Sampled Data," IEEE Journal of Selected Topics in Signal Processing, vol. 1, no. 4, pp. 575-585, Dec. 2007.

[9] R. Tibshirani, "Regression Shrinkage and Selection via the Lasso," Journal of the Royal Statistical Society. Series B (Methodological), vol. 58, 1996

[10] S. Boyd, "Distributed Optimization and Statistical Learning via the Alternating Direction Method of Multipliers," Foundations and Trends $\mathbb{R}$ in Machine Learning, vol. 3, no. 1, pp. 1-122, 2010.

[11] P. Stoica and R. L. Moses, Spectral Analysis of Signals. Upper Saddle River, N.J: Pearson/Prentice Hall, 2005. 